\title{
3.3 CAN SHORT PERIOD COMETS MAINTAIN THE ZODIACAL CLOUD ?
}

\author{
S. Röser \\ Max-Planck-Institut für Astronomie \\ Heidelberg-Königstuhl, FRG
}

The combined effects of the Poynting-Robertson drag, collisions and sputtering are destroying the interplanetary dust cloud. The mass-losses estimated by different authors reveal great discrepancies. The estimations range from $1 \mathrm{t} \mathrm{sec}^{-1}$ (purely Poynting-Robertson loss) over some $10 \mathrm{t} \mathrm{sec}^{-1}$ given by Whipple (1967) to a value of $100 \mathrm{t} \mathrm{sec}^{-1}$ which is propagated by Bandermann (1967). The latter derives this high value from the assumption that the main mass loss is due to self-collisions.

As dust sources for the Zodiacal Cloud asteroids and comets have been discussed repeatedly. This paper deals with comets and discusses the effect of radiation pressure on the trajectories of the ejected particles. We calculate the total dust production of a comet along its orbit and the percentage of dust that does not leave the solar system immediately.

The new orbital elements of the particles after their separation from the comet are calculated under the assumption that the particles have no additional velocity component at ejection. This assumption is supported by investigations of Finson and Probstein (1968), who derived ejection velocities of only about $0.2 \mathrm{~km} \mathrm{sec}^{-1}$ for comet Arend-Roland. Then the formulae for the new orbital elements $a^{\prime}$ and $e^{\prime}$ read as follows

$$
\begin{aligned}
& a^{\prime}=\frac{a \mu}{1-2 \cdot(1-\mu) \cdot\left(\frac{a}{r}\right)} \\
& e^{\prime^{2}}=1-\frac{1-e^{2}}{\mu^{2}}+\frac{2(1-\mu)}{\mu^{2}} \cdot \frac{p}{r}
\end{aligned}
$$

where $a, e, r$ are the semi-major axis, eccentricity and radius vector of the comet resp., $\mathrm{p}=\mathrm{a}\left(1-\mathrm{e}^{2}\right)$ and $\mu=1-\frac{\text { rad. pressure }}{\text { gravity }}$

We get the particle diameter using the formula

$$
1-\mu=\frac{C}{\rho d}
$$


where $\rho$ is the mass-density, $d$ the diameter of the particle and $c=$ $1.19 \times 10^{-4} \mathrm{~g} \mathrm{~cm}^{-2}$. The size-distribution function of the ejected particles was taken from Finson and Probstein (1968), who derived this distribution from measurements of Comet Arend-Roland. The distribution has its maximum at $\rho d=3 \times 10^{-4} \mathrm{~g} \mathrm{~cm}^{-2}$, a sharp cut-off to smaller values of $\rho d$ and follows a power law (exponents between -3 and -5 ) for the decay to larger values.

We have considered two cases for the law of decay of the dust production with increasing distance from the sun: dust production is proportional (I) to the brightness of the comet $\left(r^{-4}\right)$ or (II) to the flux density of the solar radiation $\left(r^{-2}\right)$. Using these laws the emission rates have been integrated along the whole orbit of the comet.

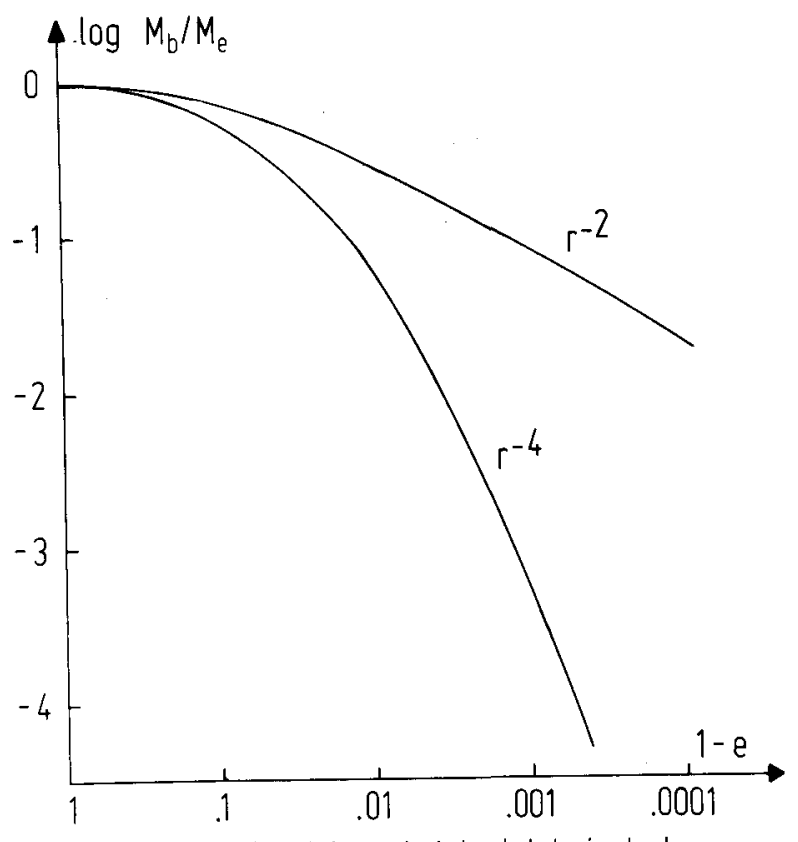

Figure 1. Ratio of bounded to total ejected mass as a function of the comet's eccentricity

In Figure 1 we have plotted the percentage of mass with $e^{\prime}<1$ against the original eccentricities for both cases. The shape of the curves is only valid under the assumptions made above about ejection velocity and size distribution. The ratio of the mass bound to the solar system de- 
creases rapidly with increasing eccentricity of the comet, though the relatively strong weight of aphelion production prevents a steeper decay. This ratio has to be taken into account if one considers the contribution of dust from long-period comets. These always produce the bound material during their passage through the aphelion parts of their orbits, that means at great distances from the Sun, and we do not know what kind of emission law can be applied there. Further, it is still an open question, whether this material can cross the barriers of the great planets and penetrate into the inner solar system.

To investigate the mass supply by short period comets, absolute mass production rates are needed. At present we know the absolute mass production only of a few long period comets. Finson and Probstein (1968) derived the mass production of Comet Arend-Roland and found a rate of $75 \mathrm{t} \mathrm{sec}^{-1}$ at the perihelion. For Comet Bennett Sekanina and Miller (1973) gave a value of some $20 \mathrm{tsec}^{-1}$. These two values agree sufficiently well using the formula

$$
-2.5 \log \mathrm{E}_{\mathrm{q}}=\mathrm{H}_{10}+10 \log \mathrm{q}
$$

where $\mathrm{E}_{\mathrm{q}}$ stands for the relative emission rate at perihelion, $\mathrm{H}_{10}$ for the comet's absolute magnitude and $q$ for its perihelion distance. The formula is equivalent to the assumption that the comet's emissivity at perihelion is proportional to its brightness there $\left(10^{-0.4 \times \mathrm{H}_{10}} \mathrm{q}^{-4}\right)$. Using this formula we calculated the perihelion mass production of the 55 short period comets of Wachmann's list (1965). So we linked the mass production of the short period comets to the production rate of two long period resp. new comets. Integrating now the emission rates along the orbits yields mean production rates for each comet.

\begin{tabular}{|l|c|c|cc|}
\hline Name & total & elliptic & total & elliptic \\
\hline Halley & 58.4 & 14.6 & 154.0 & 77.0 \\
Pons-Brooks & 23.7 & 8.1 & 62.1 & 37.3 \\
Encke & 22.6 & 16.5 & 56.7 & 48.2 \\
Blela & 12.0 & 10.1 & 28.7 & 26.7 \\
Brorsen & 11.8 & 9.3 & 29.2 & 26.3 \\
Olbers & 7.3 & 3.5 & 19.0 & 13.3 \\
All others & 12.5 & 10.7 & 27.3 & 24.4 \\
\hline Sum & 148.3 & 72.8 & 377.0 & 253.2 \\
\hline Assuned law & & & & \\
for emission & & $\mathrm{r}^{-4}$ & & \multicolumn{2}{|c|}{} \\
rate & & & \\
\hline
\end{tabular}

Table 1. The best dust producers of the short period comets for different laws of the emission rate. Masses are given in $\mathrm{kg} \mathrm{sec}{ }^{-1}$. 
In Table 1 we list the best dust producers among the short period comets. Comet Halley turns out to be the best dust source of all, but because of its high eccentricity most of the dust is lost from the solar system, whereas Encke produces a relatively higher amount of dust having elliptic orbits. All short period comets together produce not more than 72 resp. $253 \mathrm{~kg} \mathrm{sec}{ }^{-1}$ of dust remaining in the solar system. This present rate is by far too low to give an essential contribution to the Zodiacal Cloud, and we conclude that short period comets are not an important source for interplanetary dust.

\section{References}

Bandermann, L.W.; Physical Properties and Dynamics of Interplanetary Dust, University of Maryland, Technical Report No 771 (1967)

Finson, M.L. and Probstein, R.F.; A Theory of Dust Comets, ApJ 154 , 327 (1968)

Sekanina, Z. and Miller, F.D.; Comet Bennett (1970 II), Science 179 , 565 (1973)

Wachmann, A.; in "Landold - Börnstein", Zahlenwerte und Funktionen, New Series, Group VI, Vol. 1,176 (1965)

Whipple, F.; in "The Zodiacal Light and the Interplanetary Medium", ed. by Weinberg, J.L., NASA SP 150, 409 (1967) 